Proceedings of the 1999 IEEE

International Conference on Robotics \& Automation

Detroit, Michigan • May 1999

\title{
Development of a Myoelectric Discrimination System for a Multi-Degree Prosthetic Hand
}

\author{
Han-Pang Huang*, Chun-Yen Chen** \\ Robotics Laboratory, Department of Mechanical Engineering \\ National Taiwan University, Taipei 10674, TAIWAN, RO.C. \\ Tel/Fax: (886)2-23633875 \\ E-mail: hanpang 0 ccms.ntu.edu.tw \\ *Professor and correspondence addressee $\quad{ }^{* *}$ Graduate student
}

\begin{abstract}
A multiple degrees of freedom prosthetic hand, NTU Hand, was developed in our laboratory. This paper is focused on the development of a myoelectric discrimination system for a multi-degree prosthetic hand. The discrimination system uses two surface electrodes to acquire the electromyography (EMG) signal from the flexor digitorum superficialis muscle and the extensor pollicis brevis muscle. Since eight types of hand movements, such as three-jaw chuck, lateral hand, hook grasp, power grasp, cylindrical grasp, centralized grip, flattened hand and wrist flexion are often used in daily life, they are used as key movements in the discrimination system. In order to distinguish those hand movements, the techniques of variance, bias zero-crossings, autoregressive model and spectral estimation are employed for preprocessing of features. Then, an error backpropagation neural network is applied to discriminate among the feature sets. Finally, an analysis interface system based on $\mathrm{PC}$ environment is constructed to verify the idea. Besides, a 3-D graphic interface program based on programmer's hierarchical interactive graphic system (PHIGS) is applied to simulate the prosthetic movement of the NTU hand. The discrimination system can achieve a success rate of $85 \%$ for off-line test and of $71 \%$ for on-line test.
\end{abstract}

\section{Introduction}

Recently, the number of the physically handicapped has increased due to traffic accidents, workshop accidents and diseases, and most of them belong to limb losers. In order to improve the life quality, many amputees tend to use external power to operate artificial prosthesis or orthotic arms instead of cosmetic hands. For example, they may use body movements, high-pressure air or electric actuators as external power to manipulate their prosthesis. For replacing most functions that a normal man may do daily, multifunctional or multi-degree prosthesis has been proposed to satisfy this requirement $[4,13]$. However, a major problem in those researches is how can the amputee control the prosthesis more easily, directly and intuitively. In other words, it is expected that a natural feeling of control similar to that of the original limb can be realized. Since electromyography (EMG) is the electrical manifestation in the contracting muscles, it is the most simple and direct way to represent the contracting information of a muscle. Many attempt to use the EMG signal as the command to control the prosthesis $[3,5,6,17,18]$. By using EMG signals, it is essential to distinguish different limbs and hand movements from the patterns of EMG signals that are recorded at the stump.
The most widely approach is to use the pattern recognition scheme to achieve this task.

Several methods have already been reported on the EMG pattern recognition for forearm movements $[3,4,8,10,11,15,16]$. However, most of them collect EMG signals from the biceps and triceps. Their goals are almost focused on the elbow and wrist movements, such as elbow extension/flexion, wrist pronation/supination, wrist abduction/adduction, and so forth. Conversely, the prehensile postures of a hand or hand movements are seldom addressed. According to daily activities of human being and clinical survey, we believe that the prehensile functions should also be added to the multi-degree prosthetic hand. Hence, the prosthesis can be more ideal.

Consequently, we treat the prehensile postures that a person often activates daily as our design and control goals. There are a total of 87 external mechanical degrees of freedom of the upper extremity. The upper extremity consists of two main functional parts: $<1>$ the terminal prehension device (hand/wrist), $<2>$ a crane system (the rest of arm/shoulder system). For the terminal device, the basic function is to provide the proper grip for functional activities. Prehension is the primary function of that. In this study, eight types of prehensile postures are selected from $[2,12]$ for the pattern recognition. Each one of the selected postures is a typical mode in the daily activity. Besides, most of them are also identified by an article "Specifications for electro-mechanical hand" [1] which is published by the prosthetics community. The list of those postures are as follows.

$<1>$ Three-jaw chuck or palmar tripod pinch: This is used to hold small objects.

$<2>$ Lateral pinch: This is usually used to hold a key while unlocking a door. It is also named as a key grasp.

$<3>$ Hook grasp: This is used to carry items, such as books or a briefcase.

$<4>$ Power grasp: This is used to powerfully grasp heavy or relatively large objects.

$<5>$ Cylindrical grasp: It is used to grasp the large cylindrical objects.

<6> Flattened hand: In this posture, the thumb must be rotated completely out of opposition of the fingers. It is used to hold the large surface objects, such as books and plates.

$<7>$ Centralized grip or indexing hand: It is used to hold object that essentially elongates the hand distally, such as a fork, a knife or a pointer.

$<8>$ Wrist flexion. 

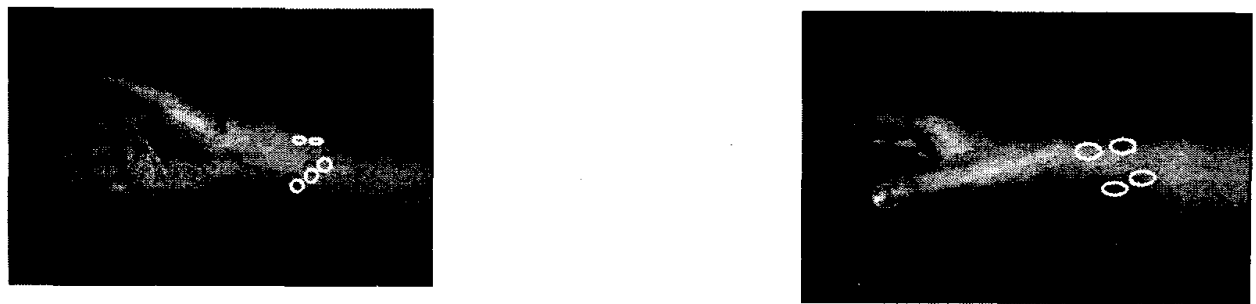

Fig.1. Placement of these two electrodes

In this paper, surface electrodes are used to generate the surface EMG. The surface EMG is stochastic in nature. It is composed of a summation of asynchronously triggered motor unit pulse trains. The differentially amplified EMG from a single muscle is characterized by a zero mean value and a variance, which is proportional to the muscle contraction level. Since the EMG signal around the distal forearm contains more information of prehensile postures than mid-forearm or any others, we focus on the amputees who belong to the below wrist disarticulation with good stump function in the paper. Nevertheless, the disarticulation level is not the most general case in the clinical diagnosis.

In order to identify the aforementioned eight hand movements, several preprocessing techniques and neural network are constructed for pattern recognition of EMG signals. The techniques of variance, bias zero-crossings, autoregressive model and spectral estimation are developed for feature preprocessing. Then, an error backpropagation neural network is applied to discriminate among the feature sets. An analysis interface system based on PC environment is also constructed to verify the idea. Besides, a 3-D graphic interface program based on programmer's hierarchical interactive graphic system (PHIGS) is applied to simulate the prosthetic movement of the NTU hand. The discrimination system can achieve a success rate of $85 \%$ for off-line test and of $71 \%$ for on-line test.

\section{Signal Preprocessing}

According to the relation between the muscle locations and the hand movement, two electrode locations are selected. The anterior side electrode is placed on the flexor digitorum superficialis. The other posterior side electrode is placed on the extensor pollicis brevis. The primary reason for selecting those locations is that muscles around those two places have significant correlation with prehensile postures.

Normal resting muscle shows no changes in potential; however, a contracting muscle gives large changes in potential. We use the IEMG (Integrated EMG) feature as the index to detect the muscle activity condition by telling the firing point of the EMG signal sequence. In this concept, a sliding window is used as the boundary for calculating the IEMG value. The calculation of the IEMG value is given by

$I E M G_{k}=\sum_{j=k-N}^{k}\left|X_{j}\right|$, For the first window

$I E M G_{k+1}=I E M G_{k}+\left(X_{k+1}-X_{k-N+1}\right), \quad$ For the subsequent update where $\mathrm{N}$ is the window size; $\mathrm{X}_{\mathbf{k}}$ is the $\mathrm{k}^{\text {th }}$ sampling data.
The detection performance depends on the size of the sliding window and the magnitude of the threshold value. A smaller size of window and less threshold magnitude can increase the sensitivity of the detecting algorithm. However, the unstable problem may be induced.

In order to overcome the unstable problem, the IEMG detection method is modified to act like a Schmitt gate. First, a mean threshold value is determined based on the rest of EMG signals. Then, two different factors are respectively applied to the rising-edge and the falling-edge of the EMG signal to form two different threshold values for detection. These two threshold values are calculated by the following formulas.

Upping-edge threshold value $=$ Mean IEMG value $\times 3.5$

Falling-edge threshold value $=$ Mean IEMG value $\times 1.5$

\section{Pattern Recognition}

A pattern recognition system can be divided into feature extraction stage, feature selection stage, and classification stage. First, the feature extraction is performed on the raw data to extract the features of the input patterns. Next, a smaller set of meaningful features that best represent the patterns are identified in the stage of feature selection. In the classification stage, a specific pattern is assigned to a specific class according to the relations that are established during the training or learning period.

The success of a pattern recognition system depends almost entirely on the choice of features representing the data sequence. The EMG signal can be represented in various forms or parameters. Different forms or parameters result in different analytical complexity and functional advantages. The algorithms for EMG feature extraction or parametric representations are described below.

\section{Traditional Parametric Features}

The traditional parametric features of EMG signals include integrated EMG variance, bias zero-crossings, slope-sign changes, waveform length and Willison amplitude parameter. They are all from the real-world processes. However, computational complexity is the major concern. All of them can be computed in real-time. For each feature $\mathrm{X}_{k}$ denotes the $k$ th sampling data in the window and $\mathrm{N}$ is the window length for computing the features.

- Integrated EMG (IEMG): This parameter is found by calculating the summation of the absolute values of EMG signals. It can be treated as a signal power estimator. It is defined as 


$$
I E M G=\sum_{k=1}^{N}\left|x_{k}\right|
$$

- Variance: This parameter is used to estimate the power density of the EMG signal. Its definition is given by

$$
\text { Var }=\frac{1}{N-1} \sum_{k=1}^{N} x_{k}^{2}
$$

- Bias Zero-Crossings (BZC): Zero-crossings is the number of times that the signal passes the zero amplitude axis. This parameter is used to get the rough property in frequency domain. In this paper, for preventing influence from background disturbance, a bias is added to the calculation of the zero-crossings value to from the bias zero-crossings. It is calculated as

$$
\begin{aligned}
& Z C=\sum_{k=1}^{N} \operatorname{sgn}\left(\left(X_{k}-0.4\right) \times\left(X_{k-1}-0.4\right)\right) ; \\
& \operatorname{sgn}(x)=\left\{\begin{array}{l}
1, \text { if } \quad x>0 \\
0, \text { otherwise }
\end{array}\right.
\end{aligned}
$$

- Slope-Sign Change: This is another parameter to represent the frequency information. As mentioned above, a suitable value shall also be included to reject the disturbance effect. The criterion for the parameter selection is defined by the consecutive samples as

$[\mathrm{X}(\mathrm{k})-\mathrm{X}(\mathrm{k}-1)] \times[\mathrm{X}(\mathrm{k})-\mathrm{X}(\mathrm{k}+1)] \geq 0.003$

If the condition is satisfied, the slope-sign change value is increased.

- Waveform Length: This is a parameter that can estimate the complexity of the EMG waveform. The calculation is defined as

$$
\text { wavelength }=\sum_{k=1}^{N}\left|x_{k}-x_{k-1}\right|
$$

- Willison Amplitude (WAMP): This parameter is used to count the number of times that the signal amplitude exceeds a predefined threshold. It is an indicator of firing of MUAP and therefore an indicator of muscle contraction level. The definition is as

$$
\begin{aligned}
\text { WAMP } & =\sum_{i=1}^{N} f\left(\mid X_{i}-X_{i-1}\right) ; \\
f(x) & = \begin{cases}1, & \text { if } \\
0, \text { otherwise }\end{cases}
\end{aligned}
$$

\section{Autoregressive (AR) Parametric Model}

Graupe et al. [5,6] showed that, for stationary Gaussian statistics, the EMG signal could be modeled as an autoregressive (AR) time series. Basically, it is a kind of linear prediction. According to spectral analysis $[7,9,14]$, an AR series belongs to an all-pole model. The detailed spectral characters will be described later. The benefits of the AR parametric model are that the EMG signal can be represented by model parameters without the original waveform data. Hence, the amount of data can be enormously reduced and the specific features of signal can be reinforced. An autoregressive model is defined by

$$
y_{k}=-\sum_{i=1}^{N} A_{i} \times y_{k-i}+w_{k}
$$

where $A_{i}$ is the $A R$ coefficients; $N$ is the model order; $y_{k}$ is the output sequence of the AR model; $y_{k-1}$ is the input sequence and $w_{k}$ is the white noise signal. The previous research $[5,9,14]$ showed that a model of order four is adequate for modeling an EMG signal.

Spectral Non-parametric Model

The classical spectral estimation is the power spectral density (PSD) function. It is a non-parametric modeling method in frequency domain. The PSD sequence is divided into six frames to generate the spectral features. The reason for selecting those frequency frames is according to the signal's Spectrogram.

For catching the time domain information from the frequency domain and reducing the data dimension, the ranges for creating the spectral parameters should be properly chosen. We select the frequency regions that EMG signals fluctuate very often over time. Consequently, the spectral characteristic in temporal like Short-Time Fourier Transform [7] are directly included in those timedependent spectral parameters. The final result is given Table 1.

\begin{tabular}{|c|c|}
\hline \multicolumn{1}{|c|}{ Table 1 Frequency range of the six frames } \\
\hline $\begin{array}{c}\text { Number } \\
1\end{array}$ & Spectral Range \\
\hline 2 & $30 \sim 60 \mathrm{~Hz}$ \\
\hline 3 & $60 \sim 75 \mathrm{~Hz}$ \\
\hline 4 & $75 \sim 90 \mathrm{~Hz}$ \\
\hline 5 & $90 \sim 120 \mathrm{~Hz}$ \\
\hline 6 & $120 \sim 180 \mathrm{~Hz}$ \\
\hline
\end{tabular}

Basically, the autoregressive (AR) model is an allpole filter. Due to the lack of zeros, the AR model is difficult to model "holes " in the spectrum. This defect can be compensated by a very high order AR model. However, an AR model always gives a smoother spectral curve in contrast to classical spectral estimation [14].

Feature Selection Stage

In this paper, we apply the Davies-Bouldin (DB) cluster separation measure method as the index to achieve the selection task [17]. DB index is the parametric estimation that can directly addresses the issue of the cluster separability in the feature space. The index is used to tell the different features' clustering performance. It is defined based on the cluster-to-cluster similarity as

$$
R_{i j}=\frac{\left(S_{i}+S_{j}\right)}{D_{i j}}
$$

where $S_{i}$ and $S_{j}$ are the dispersions of the $i^{\text {th }}$ and $j^{\text {th }}$ clusters respectively, and $D_{i j}$ is the distance between their mean values, as shown in Figure 10. The dispersion of a cluster is defined as

$$
S_{i}=\left\{\frac{1}{N_{i}} \sum_{j \neq i}^{N_{N}}\left(y_{i}-m_{i}\right)^{T}\left(y_{j}-m_{i}\right)\right\}^{1 / 2}
$$

where $N_{i}$ is the number of members of cluster $C_{i} ; y_{i}$ is the $i^{\text {th }}$ input pattern vector; and $m_{i}$ is the mean vector of cluster $i$. The distance $D_{i j}$ between two clusters is calculated by the Euclidean distance norm as

$$
D_{i j}=\left\{\left(m_{i}-m_{j}\right)^{T}\left(m_{i}-m_{j}\right)\right\}^{4 / 2}
$$

In this paper, the calculation of DB index is different from the original method. It is obtained through averaging two of the worst case separation of one cluster from the other instead of only one worst separation, i.e.

$$
D B=\frac{1}{2 K} \sum_{i=1}^{K}\left[\max _{l \neq j}\left(R_{i j}\right)+s u b \max _{i \neq j}\left(R_{i j}\right)\right]
$$


In essence, the DB index reports how badly the clusters overlay their nearest neighbors. A lower value of the DB index implies a higher degree of cluster separability. By averaging the two worst cases, the separation measurement is more objective.

\section{Classification Stage (Artificial Neural Network)}

For distinguishing the EMG patterns, a backpropagation (BP) neural network is applied to EMG features. A multi-layer perceptron with error backpropagation algorithm has been successfully applied to some difficult and nonlinear problems in diverse domains. An error back-propagation neural network with one hidden layer and one output layer is developed in this paper. The structure of the network is based on one-classone-network (OCON) concept and the transfer functions for hidden layer neurons and output layer neurons are all nonlinear sigmoid functions. The number of neurons in the hidden layer is determined by the following relation

$$
\text { HidenNum }=\left(\text { InputNum }+\log _{2} \text { OutputNum }\right) / 2
$$

\section{Implementation}

Since the PC environment is an open system, it is easy to change or modify the algorithm. We first develop the PC-based implementation to verify the concept. The whole schematic diagram of the system is shown in Figure 2.

In the system, there are three major parts: two channel measurements with filter circuit, a personal computer (PC) and a host workstation. The filter circuits are used to preprocess the EMG signals. The PC that contains an $\mathrm{AD} / \mathrm{DA}$ card is used to acquire the signal and perform the analysis task. The host workstation is used to simulate the prosthesis dynamics. The communication between the PC and host workstation is achieved by RS232 port.

The flowchart of the system kernel is shown in Figure 3. This flowchart is run through when the system is operated in the real-time model. The system is operated in two modes: data analysis mode and analysis processing mode. When the system is in the data-analysis mode, the flow can be terminated at any stage depending on what kind of event is triggered in the user interface.

In the analysis processing, a sliding window that contains 64 points' data is first used to detect the muscle activity. Whenever the firing-detecting criterion is satisfied, the data collection stage is enabled and 512 points' data is collected to prepare for the next step. In the mean time of data collection, the firing detector is still processing, and the data-collection task will be reset when the firing criterion is not satisfied. Then, the 512-point data is sent to the feature creation stage. In the feature creation stage, several methods are used to create 108 features for each movement. Then the neural network is used to distinguish the result, and the artificial prosthetic simulator is activated.

In this paper, we use several feature-extraction techniques to create the pattern feature. Before applying the technique, two channels of EMG signals are collected from the limb with $1 \mathrm{KHz}$ sampling rate. For each channel, 512-point data is recorded from the firing point in each hand movement. Then they are divided into four frames to generate the temporal information. The concept is shown in Figure 4. Thus, several features can be created based on different frames and total collected windows. The created feature parameters are listed in Table2.

The 3D graphic interface is designed for simulating the seventeen joints dynamic relationship of the NTU hand. The simulation is performed on a SUN sparc 10 Workstation under the $\mathrm{X}$ environment. It is constructed by PHIGS (Programmer's Hierarchical Interactive Graphics System) 3D graphic library. In this study, the PHIGS interface is used to play the role as an actual prosthesis. Consequently, a virtual action can reflect the EMG classified result. The correlation between each hand posture and 17-joint angles are established by experiment, then the outcome values are recorded into a table. The simulated hand movements are shown from Figure 5 to Figure 12.

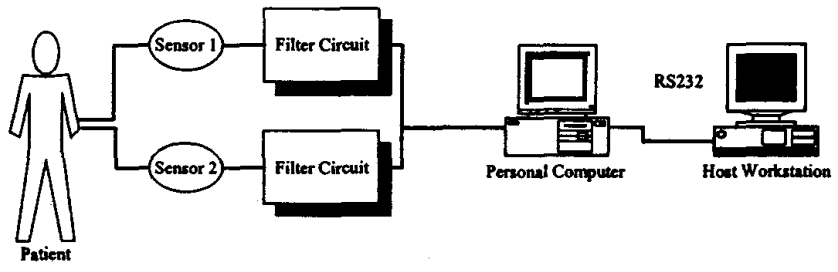

Fig. 2. PC-based analysis system

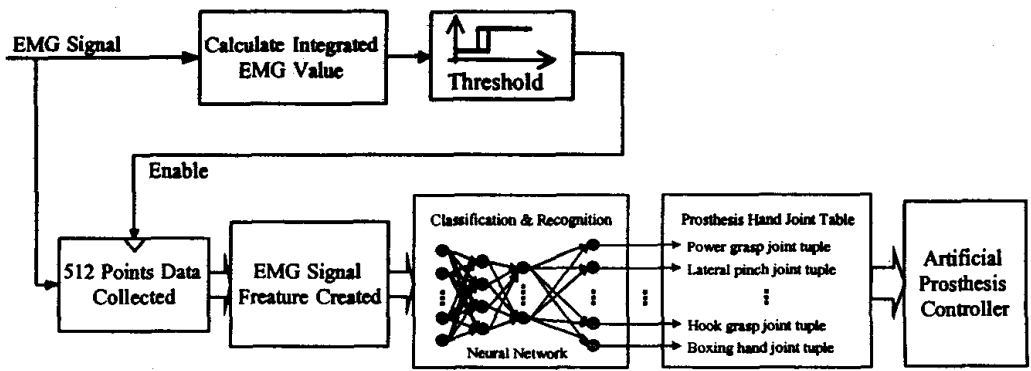

Fig.3. System kernel flow chart 


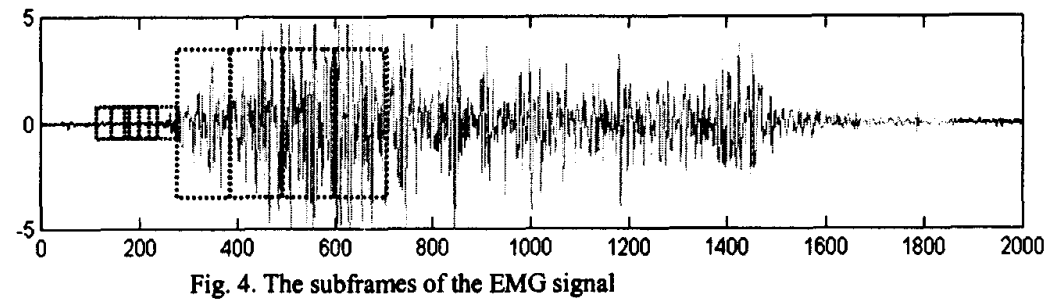

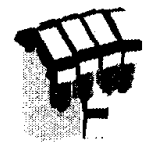

Fig. 5. Power grasp Fig. 6. Hook grasp
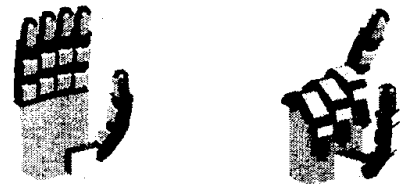

Fig.9. Flattened hand Fig.10. Centralized grip

\section{Results and Discussions}

In order to demonstrate the system performance, three data sets are used to form the off-line analysis result. Those data sets come from two normal subjects. Two data sets are collected from the first subject at different times with a slightly different electrode locations. The third data set is collected from another subject. All data sets contain two-channel EMG signal of eight types of hand movements.

\section{Off-line Analysis Results}

The comparison index among those pattern feature distributions is based on the Davies-Bouldin (DB) cluster separation measurement. The result is given in Figure 13.

From the results, Variance, Wave-Length and IEMG have better cluster separability than others, and most of their $\log$ scale values also show better performance than linear scale values. In addition, AR model parameters for a long window of data give better cluster separability for a short one. Also, the first two parameters of AR model have better cluster separability than the last two.

Table 2. List of created features for each hand movement

\begin{tabular}{|c|c|c|c|}
\hline Parameter Title & $\begin{array}{c}\text { Parameter } \\
\text { Number }\end{array}$ \\
\hline $4^{\text {th }}$ order AR model & $4 \times 2$ \\
\hline $\begin{array}{c}4^{\text {th }} \text { order Cepstrum } \\
\text { parameters }\end{array}$ & $4 \times 2$ \\
\hline $\begin{array}{c}\text { Zero-Crossings } \\
\text { Number }\end{array}$ & $1 \times 2$ \\
\hline IEMG \& Variance & $2 \times 2$ \\
\hline Wave Length & $1 \times 2$ \\
\hline Parameter Title & $\begin{array}{c}\text { Number of } \\
\text { Parameters }\end{array}$ \\
\hline $\begin{array}{c}\text { Wth order AR } \\
\text { model }\end{array}$ & $4 \times 2$ \\
\hline $\begin{array}{c}\text { Spectral } \\
\text { parameters }\end{array}$ & $6 \times 2$ \\
\hline Slope-Sign Changes & $1 \times 2$ \\
\hline \multicolumn{2}{|c|}{ Total Number $=\{(4+4+1+2+1+1+1)+[(4+6) \times 4]\} \times 2=108$} \\
\hline \multicolumn{2}{|c|}{} \\
\hline
\end{tabular}

In order to find the balance between the performance and system response speed for the application, a suitable data window length for the analysis should be properly chosen. A comparison of performance for different data lengths is shown in Figure 14. The results verify that a long data length will ensure better cluster separability, even though the length seems to be short in a real-time application. From the result, the length for on-line
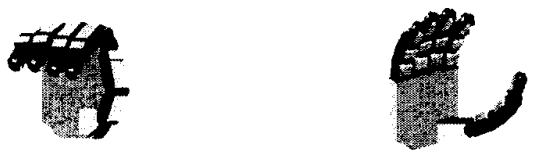

Fig. 7. Free hand Fig. 8. Lateral pinch
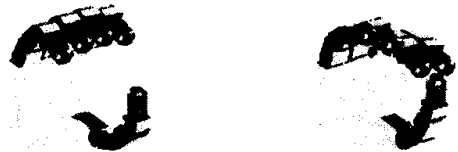

Fig.11. Three-jaw pinch Fig.12. Cylindrical grasp

application is chosen as 256 points $(0.256 \mathrm{msec})$.

In order to get the best performance, the best features which are determined at the previous stage are combined together to reinforce the whole clustering performance. From the results, two combinations of features are suggested. One is the combination of IEMG, Variance, Wave length and WAMP. The other is the combination of IEMG Variance, Wave length, WAMP, $\mathrm{BZC}$ and $2^{\text {nd }}$ order $\mathrm{AR}$ model parameters. These two combined feature set are used in the neural network classification engine to test the results.

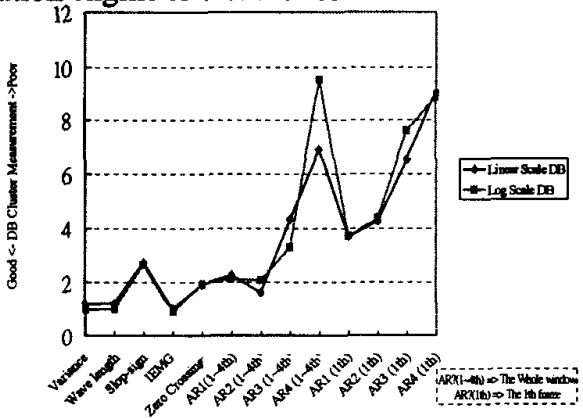

Fig.13. Cluster separation measurement for two-channel different parameters in both linear and log scale (Data I)

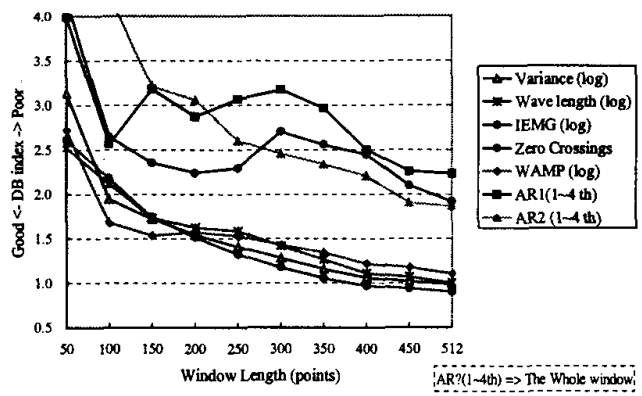

Fig.14. Window length vs. different feature cluster separation measurement

In the actual classification performance analysis, three data sets are individually applied to the neural 
network. Before applying the network, half of each data set is used to train the network. Then, the other half data set is used to test the network performance. Alternately, the roles of testing set and training set are changed and the network is applied again. Finally, the two testing results are averaged to form the final result. The result of the testing is listed in Table 3 and Table 4 . Table 3 uses those 4 kinds of pattern features for recognition, while Table 4 uses 6 kinds of parameters as features.

On-line Testing Results

Besides the off-line demonstration, an on-line testing is also applied to verify the scheme. The result ant performance is listed in Table 5

\section{Conclusion}

The discrimination of eight kinds of prehensile postures has been successfully justified by using the forearm EMG signals. In this study, we also demonstrated that the combination of IEMG Variance, WAMP, Wave length, zero-crossings and $2^{\text {nd }}$ order $\mathrm{AR}$ parameters has the best performance for the pattern recognition of those postures. The spectral estimation method, short-period spectral parameters, and short-period AR model show poor result for this rapid movement discrimination. In practice, after proper learning, the identified success rate for neural network is about $85 \%$ in off-line testing and $71 \%$ in online testing.

In the future, a recurrent neural network method, such as unsupervised learning rule can be included in the prosthesis system. Then, the system can dynamically adapt to the gradual changes of the EMG patterns induced from muscle fatigue and sweat. In addition, the previous training data need not be regenerated.

Table 3. The correct rate of using IEMG, Variance, Wave Length and WAMP as features for postures classification

\begin{tabular}{|c|c|c|c|}
\hline Data Set & $\begin{array}{c}\text { Testing Correct } \\
\text { Rate }\end{array}$ & $\begin{array}{r}\text { Testing Correct } \\
\text { Rate }\end{array}$ & $\begin{array}{c}\text { Average } \\
\text { Correct Rate }\end{array}$ \\
\hline Data I & $82.1 \%$ & $92.5 \%$ & $87.3 \%$ \\
\hline Data II & $82.1 \%$ & $87.3 \%$ & $84.7 \%$ \\
\hline Data III & $79.2 \%$ & $86.3 \%$ & $82.7 \%$ \\
\hline
\end{tabular}

Table 4. The correct rate of using IEMG, Variance, Wave Length, WAMP, $\mathrm{BZC}$ and $2^{\text {nd }}$ order AR as features for postures classification

\begin{tabular}{|c|c|c|c|}
\hline Data Set & $\begin{array}{c}\text { Testing Correct } \\
\text { Rate }\end{array}$ & $\begin{array}{c}\text { Testing Correct } \\
\text { Rate }\end{array}$ & $\begin{array}{c}\text { Average Correct } \\
\text { Rate }\end{array}$ \\
\hline Data I & $89.7 \%$ & $93.8 \%$ & $91.7 \%$ \\
\hline Data II & $92.4 \%$ & $91.1 \%$ & $91.8 \%$ \\
\hline Data III & $83.3 \%$ & $82.5 \%$ & $82.9 \%$ \\
\hline
\end{tabular}

Table 5. On-line testing result

\begin{tabular}{|c|c|}
\hline Movement Name & Correct Rate \\
\hline Three-jaw chuck & $46.4 \%$ \\
\hline Lateral pinch & $73.6 \%$ \\
\hline Hook grasp & $81.8 \%$ \\
\hline Power grasp & $55 \%$ \\
\hline Cylindrical grasp & $70.0 \%$ \\
\hline Flattened hand & $100.0 \%$ \\
\hline Centralized grip & $40.8 \%$ \\
\hline Wrist flexion & $100.0 \%$ \\
\hline Average success rate & $71.0 \%$ \\
\hline \multicolumn{2}{|c|}{ References }
\end{tabular}

References

[1] D.J. Atkins and R.H. Meier, Comprehensive Management of the Upper-Limb Amputee, London: Springer-Verlag, 1988.

[2] A.M.R. Agur, Grant's Atlas of Anatomy, ninth edition, London: Williams \& Wilkins, 1991.

[3] P.C. Doerschuk, D.E. Gustafson and A.S. Willsky, "Upper extremity Limb Function Discrimination Using EMG Signal analysis," IEEE Transactions on Biomedical Engineering, Vol. BME-30, No. 1, pp.
18-28, January 1983.

[4] K.A. Farry, I.D. Walker and R.G Baraniuk, "Myoelectric Teleoperation of a Complex Robotic Hand," IEEE Transactions on Robotics and Automation, Vol. 12, No. 5, pp. 775-788, 1996.

[5] D. Grupe and W.K. Cline, "Functional Separation of EMG Signals via ARMA Identification Methods for Prosthesis Control Purpose," IEEE Transactions on Systems, Man and Cybernetics, Vol. SMC-5, No. 2, pp. 252-259, March 1975.

[6] D. Graupe, J. Magnussen and A.A. Beex, "A Microprocessor System for Multifunctional control of Upper-Limb Prosthesis via Myoelectric Signal Identification," IEEE Transactions on Automatic Control, Vol. AC-23, No. 4, pp. 538-544, August 1978.

[7] B. Hannaford and S. Lehman, "Short Time Fourier Analysis of the Electromyogram: Fast Movements and Constant Contraction," IEEE Transactions on Biomedical Engineering, Vol. BME-33, No. 12, pp. 1173-1181, December 1986.

[8] M.F. Kelly, P.A. Parker and R.N. Scott, "The Application of Neural Networks to Myoelectric Signal Analysis: A Preliminary Study," IEEE Transactions on Biomedical Engineering, Vol. 37, No. 3, pp. 221-230, March 1990.

[9] W.J. Kang, J.R. Shiu, C.K. Cheng, H.W. Tsao and T.S. Kuo, "The Application of Cepstral Coefficients and Maximum Likelihood Method in EMG Pattern Recognition," IEEE Transactions on Biomedical Engineering, Vol. 42, No. 8, pp. 777-785, August 1995.

[10] M.F. Kelly, P.A. Parker and R.N. Scott, "Myoelectric Signal Analysis Using Neural Networks," IEEE Engineering in Medicine \& Biology Magazine, pp. 61-64, March 1990.

[11] K. Kuribayashi, K. Okimura, and T. Taniguchi, "A Discrimination System Using Neural Network for EMG-Controlled Prosthesis," IEEE International Workshop on Robot and Human Communication, pp. 63-68, 1992.

[12] I.A. Kapandji, The Physiology of The Joints - Vol. 1: Upper Limbs, 2nd ed., N.Y.: Churchill Livingstone, 1983.

[13] L.R. Lin and H.P. Huang, "Mechanism Design of A New Multifingered Robot Hand," IEEE Intl. Conf. on Robotics and Automation, pp. 1471-1476, 1996.

[14] S.L. Marple, Digital Spectral Analysis With Applications, New Jersey: Prentice-Hall, 1987.

[15] C.S. Pattichis, C.N. Schizas and L.T. Middleton, "Neural Network Models in EMG Diagnosis," IEEE Transactions on Biomedical Engineering, Vol. 42, No. 5, pp. 486-496, May 1995.

[16] G.N. Saridis and T.P. Gootee, "EMG Pattern Analysis and Classification for a Prosthetic Arm," IEEE Transactions on Biomedical Engineering, Vol. BME-29, No. 6, pp. 403-412, June 1982.

[17] M. Zardoshti-Kermani, B.C. Wheeler, K. Badie and R.M. Hashemi, "EMG Feature Evaluation for Movement Control of Upper Extremity Prostheses," IEEE Transactions on Rehabilitation Engineering, Vol. 3, NO. 4, pp. 324-333, December 1995. 